\title{
Identificando propriedades essenciais de registros eletrônicos de saúde
}

\section{Identifying the essential properties of electronic health records}

\author{
Elisa Tuler de Albergaria ${ }^{1}$, Marcello Peixoto Bax², Raquel Oliveira Prates ${ }^{3}$, Zilma Silveira Nogueira Reis ${ }^{4}$ \\ ${ }^{1}$ Universidade Federal de Minas Gerais - UFMG, Belo Horizonte, MG, Brasil; Universidade Federal de São João del Rei - UFSJ, São João del Rei, MG, Brasil \\ 2 Escola de Ciência da Informação, Universidade Federal de Minas Gerais - UFMG, Belo Horizonte, MG, Brasil \\ ${ }^{3}$ Departamento de Ciência da Computação, Universidade Federal de Minas Gerais - UFMG, Belo Horizonte, MG, Brasil \\ ${ }^{4}$ Faculdade de Medicina, Universidade Federal de Minas Gerais - UFMG, Belo Horizonte, MG, Brasil
}

Autor para correspondência/Mail to: Elisa Tuler de Albergaria (etuler@gmail.com)

Financiamento/Funding: Coordenação de Aperfeiçoamento de Pessoal de Nível Superior - Capes

Recebido/Submitted: 17 Jan. 2016; Aceito/Approved: 19 Abr. 2016

Copyright (c) 2016 Albergaria et al.. Todo o conteúdo da Revista (incluindo-se instruções, política editorial e modelos) está sob uma licença Creative Commons Atribuição-NãoComercial-Compartilhalgual 3.0 Não Adaptada. Ao serem publicados por esta Revista, os artigos são de livre uso em ambientes educacionais, de pesquisa e não comerciais, com atribuição de autoria obrigatória. Mais informações em http://ojs.c3sl.ufpr.br/ojs2/index.php/atoz/about/submissions\#copyrightNotice.

\begin{abstract}
Resumo
Introdução: Inserido no desafio de possibilitar a interação de profissionais da saúde com prontuários eletrônicos baseados na Norma ISO 13606 permitindo a personalização de interface ao mesmo tempo que se preserva a estrutura e padronização dos Sistemas de Registros Eletrônicos de Saúde (S-RES), o presente recorte objetiva caracterizar propriedades essenciais de S-RES - flexibilidade, padronização e estrutura e facilidade de interação - analisando-se três destes sistemas.

Método: Utilizou-se o método de Design Science Research que busca gerar conhecimento teórico a partir da resolução de um problema prático (identificado no contexto da investigação mais ampla). Mais especificamente, no objetivo explicitado neste recorte, efetivou-se uma revisão de literatura sobre os requisitos existentes e dificuldades dos usuários dos sistemas S-RES, seguida de um levantamento e caracterização das propriedades essenciais dos S-RES e do estabelecimento de critérios de análise. O método de inspeção completa a proposta metodológica, visto que as questões (que indicam os critérios) foram apresentadas a dois especialistas para a análise dos Sistemas.

Resultados: No recorte proposto, as propriedades levantadas como essenciais dos sistemas foram caracterizadas e analisadas em três S-RES. Confirmou-se a importância das propriedades essenciais destes tipos de sistemas, ainda que verifique a dificuldade de serem atendidas em um mesmo sistema.

Conclusão: Os desafios postos aos S-RES não são pontuais ou mesmo de um contexto de uso. Como trabalho futuro propõe-se a criação de um modelo de interface extensível de interface para sistemas de Registro Eletrônico de Saúde baseados na ISO 13606.
\end{abstract}

Palavras-chave: Informática em Saúde; Registros eletrônicos de saúde; Interface humano-computador; Propriedades de sistemas informatizados

\begin{abstract}
Introduction: Inserted in the challenge of enabling the interaction of health professionals with electronic medical records - based on ISO 13606 - as well as allow them to customize interfaces (although preserving the structure and standardization of Electronic Health Records Systems (S-RES), the objective of this cutout paper is to characterize the essential properties of S-RES - flexibility, standardization and structure and ease of interaction - by analyzing three of these systems.

Method: It was used the Design Science Research method that seeks to generate theoretical knowledge as solving a practical problem (identified in the context of a broader research). More specifically, the goal detailed in this cutout, demanded a literature review of the existing requirements and difficulties of S-RES users, followed by a survey and characterization of the essential properties of the S-RES and the establishment of assessment criteria. The inspection method completes the methodological proposal, since the questions (which indicate the criteria) were presented to two experts as a basis for the analysis.

Results: In the proposed cutout, the essential properties of the systems were raised, characterized and analyzed in three S-RES. It was confirmed the importance of the essential properties of these types of systems, although hard to find in a combined way on a single system.

Conclusion: The challenges posed to the S-RES are not confined to an approach or even to a context of use. As future work it is propose the creation of an extensible interface model for Electronic Health Record systems based on ISO 13606.
\end{abstract}

Keywords: Health informatics; Electronic health records; Human-computer interface; Properties of information systems

\section{INTRODUÇÃO}

A obtenção, registro e disponibilização eletrônica de informações sobre a saúde das pessoas tem adquirido significativa importância tanto para a prestação do cuidado individual à saúde do paciente, como para análises epidemiológicas de populações, subsidiando a organização e a gestão em saúde (Detmer, Bloomrosen, Raymond, \& Tang, 2008; Hillestad et al., 2005). Esses dados, resultantes da assistência nos vários níveis de atenção à saúde, são de natureza diversa, tais como: textos, imagens, sinais biológicos originados de resultados de exames, prescrições, histórias e diagnósticos dos encontros clínicos, assistência multiprofissional, internações, entre outros (Marin \& Azevedo, 2003). Na maioria das vezes esses dados ficam armazenados nos diversos 
estabelecimentos em que uma determinada pessoa recebe atendimento durante toda a vida. Num cenário ideal, os dados de saúde, respeitado o direito ao sigilo pessoal, deveriam ser compartilhados nos diferentes ambientes.

Entretanto, armazenar e compartilhar os dados dos pacientes de forma eficiente, segura e completa não é uma tarefa trivial, dada a enorme variedade de informações envolvidas e diversidade de sistemas eletrônicos (Busato, 2015). Uma das soluções propostas surge com o conceito de Registro Eletrônico de Saúde (RES), seguido da necessidade de serem criados Sistemas de Registros Eletrônicos de Saúde (S-RES). Trata-se de iniciativa para que as informações sobre a saúde das pessoas fiquem armazenadas eletronicamente, ao longo da vida e que estejam acessíveis por usuários autorizados, no momento oportuno da prestação do cuidado (International Organization for Standardization [ISO], 2005). Esses sistemas são importantes e ousados, mas difíceis de serem desenvolvidos devido à complexidade de todo contexto de uso. Existem diferentes formas de se registrar dados assistenciais nos vários cenários de atendimento, as terminologias existentes, as especialidades médicas com diferentes demandas e particularidades e ainda a restrita utilização de padrões indispensáveis para troca de informações.

Um caminho para solucionar esses problemas envolve propor padronizações para apoiar o desenvolvimento de sistemas de informação em saúde interoperáveis ${ }^{1}$. Um dos padrões existentes é o OpenEHR ${ }^{2}$, especificação aberta para a construção de S-RES. O OpenEHR, assim como a Norma ISO 13606 (nele inspirada), propõe uma modelagem de dois níveis: a) nível do conhecimento; b) nível de informação (Beale, 2002). O nível de informação (também chamado de modelo de referência) define como as informações clínicas serão armazenadas. Já o nível de conhecimento (também chamados de arquétipos) envolve a modelagem clínica, feita pelos profissionais da saúde. A validação semântica na norma ISO 13606 ocorre com o modelo de arquétipos (Maia, 2015). O objetivo da modelagem de dois níveis é desvincular os conceitos clínicos dos dados em si, criando um sistema mais dinâmico e robusto, de manutenção e evolução mais simples. Além disso, utilizando esse padrão contribui-se para interoperabilidade entre sistemas S-RES.

Entretanto, desenvolver sistemas baseados em padrões, mas que sejam adequados às necessidades específicas dos usuários não é trivial. Esse artigo é parte de um projeto maior, pautado no seguinte desafio: como possibilitar a interação por usuários finais, profissionais da saúde, em prontuários eletrônicos baseados na Norma ISO 13606, permitindo que os mesmos possam personalizar sua interface mantendo estrutura e padronização dos sistemas? Nesse contexto, é objetivo aqui caracterizar propriedades essenciais desses tipos de sistemas (Padronização e Estrutura, Facilidade de Interação e Flexibilidade) no sentido de auxiliar no desenvolvimento dos mesmos. Para se atingir este objetivo três sistemas foram analisados de acordo com essas características.

\section{METODOLOGIA}

Simon (1996) apresenta o conceito de Design Science, destacando a importância de uma ciência que reflete sobre artefatos para resolver problemas. Nesse contexto, Wieringa (2009) distingue dialeticamente problemas práticos e teóricos, sem, contudo, separá-los. Um problema prático é a diferença que há entre a forma como o mundo é experimentado pelo(a) pesquisador(a) e como ele(a) gostaria que fosse. Porém, todo problema prático envolve problemas teóricos e práticos secundários de forma imbricada (Bax, 2013).

A pesquisa aqui apresentada visa contribuir com um problema prático mais amplo que envolve diversas etapas (Figura 1). Como resultados das etapas (1), (2) e (3) - e no escopo pretendido - verificou-se que há dificuldades para que os profissionais da saúde atuem ativamente na modelagem dos conceitos clínicos, utilizando ferramentas computacionais específicas, o que normalmente exige conhecimento avançado em informática e, mais especificamente, no padrão adotado (Albergaria, Bax, \& Prates, 2013; Albergaria, Bax, Prates, \& Rocha, 2014a, 2014b). Esse tem sido um impedimento frequente para a participação mais efetiva destes profissionais, ficando a modelagem muitas vezes sem as contribuições indispensáveis daqueles que dominam os conceitos do domínio e que são os futuros utilizadores dos sistemas.

O objetivo e resultados apresentados neste artigo se situam no contexto da etapa (4), Nesta, realizaram-se atividades visando caracterizar o modelo conceitual. Em um primeiro momento foi feita uma revisão de literatura sobre os requisitos existentes e dificuldades dos usuários dos sistemas S-RES. A segunda atividade consistiu em levantar e caracterizar as propriedades essenciais dos S-RES (Flexibilidade, Padronização e Estrutura e Facilidade de Interação). Posteriormente, critérios de análise foram estabelecidos (apresentados na Seção Questões de análise), assim como a escolha dos sistemas a serem analisados. Efetivou-se a análise em três sistemas existentes e utilizados tanto no Brasil quanto no exterior, ilustrando a dificuldade em se atender a esses requisitos.

\footnotetext{
${ }^{1} \mathrm{O}$ IEEE define a interoperabilidade como a capacidade de dois ou mais sistemas ou componentes trocarem informações e usarem as informações que tenham sido trocadas. (http://www.en13606.org/the-ceniso-en13606-standard/semantic-interoperability.

${ }^{2}$ http://www.openehr.org/.
} 
Problema prático: Problema de projeto - Como possibilitar a interação por usuários finais, profíssionais da saúde, em Sistemas RES baseados na Norma ISO 13606, permitindo que os mesmos possam personalizar sua interface mantendo estrutura e padronização dos sistemas?

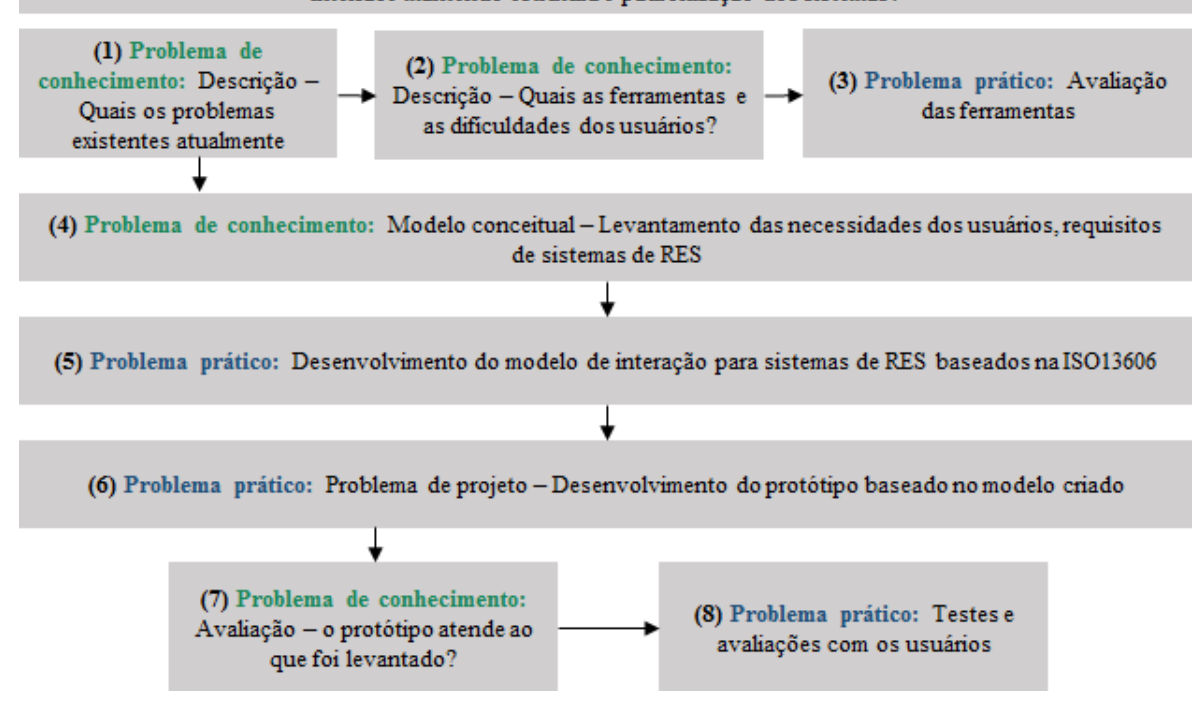

Figura 1. Decomposição do problema prático dessa pesquisa separado em etapas. Fonte: os autores (2016).

\section{TRABALHOS RELACIONADOS}

Os requisitos dos Sistemas de Registros Eletrônicos de Saúde têm sido tema de pesquisa e debate em diferentes aspectos. Um amplo estudo sobre as funcionalidades para S-RES na atenção primária pode ser encontrado em Busato (2015), por exemplo. Também é possível encontrar vários documentos publicados por entidades nacionais e internacionais que abordam funcionalidades dos S-RES (Certification Commission for Health Information Technology [CCHIT], 2011; Healthcare Information and Management Systems Society [HIMSS], 2003; Sociedade Brasileira de Informática em Saúde [SBIS], 2013).

Entretanto, além de documentos gerais, é possível encontrar trabalhos com aspectos específicos. A interface dos sistemas RES, por exemplo, é uma das preocupações, visto que o sucesso dos sistemas depende do uso efetivo por parte dos usuários. Clarke et al. (2013), por exemplo, identificaram quatro problemas comuns em S-RES: má exibição de informações, sobrecarga cognitiva, problemas de navegação, e aspectos relacionados ao fluxo de trabalho. Eles descobriram que estas questões frustram os médicos, causam erros, comprometem a interação médico paciente e a aceitação dos usuários em relação aos sistemas. Ainda buscando entender melhor os fatores que contribuem para a má usabilidade, Ratwani, Fairbanks, Hettinger, e Benda (2015) apresenta o resultado de uma pesquisa feita em 11 empresas de desenvolvimento de S-RES com o objetivo de analisar os desafios que as equipes enfrentam durante o desenvolvimento. Zahabi, Kaber, e Swangnetr (2015) efetua uma revisão na literatura de artigos relacionados à aspectos de usabilidade e segurança em sistemas de registros médicos e propõe um conjunto de diretrizes focadas no diagnóstico e documentação do processo feito pelos médicos.

A segurança dos dados, destacada por Zahabi et al. (2015), foi foco de atenção também em outros estudos. Segundo Abrahão (2003), o RES deve atender aos requisitos essenciais de integridade, autenticidade, disponibilidade e privacidade da informação. Em Bayer, Santelli, e Klitzman (105) é apresentado um novo desafio, a confidencialidade e acesso de dados de saúde de adolescente pelos seus pais. A utilização de padrões em Sistemas de RES também é abordada. Em Alves (2015), por exemplo, apresenta-se um estudo de caso do padrão OpenEHR para uma aplicação móvel de Registro Eletrônico de Saúde para uma empresa do ramo de atendimento de emergências médicas. Além destes, o estudo de Bodenreider (2004) aborda a interoperabilidade terminológica entre as aplicações médicas, tal como a UMLS (Unified Medical Language System).

Outro aspecto relevante abordado refere-se às características específicas dos S-RES. Alguns trabalhos defendem que um RES deve ser desenvolvido para atender as características de um setor específico, de modo que possa abranger todas as funcionalidades requeridas. Spooner (2007), por exemplo, especifica uma determinada especialidade, detalhando funcionalidades de sistemas para pediatria.

Entretanto, o presente trabalho aborda características que não são encontradas de forma conjunta em um único S-RES, ainda que essenciais para S-RES multi-especialidade. Ao contrário da necessidade de identificar requisitos de cada especialidade, a propriedade "flexibilidade" visa possibilitar a criação de um sistema geral que possa ser personalizado de acordo com as necessidades de cada usuário especialista. As propriedades "padronização" e "estrutura" visam permitir a interoperabilidade entre os sistemas; e a "facilidade de interação" é uma preocupação importante para que o sistema seja efetivamente usado. 


\section{O REGISTRO ELETRÔNICO DE SAÚDE}

O prontuário eletrônico é um meio físico, um repositório onde todas as informações de saúde, clínicas e administrativas, ao longo da vida de um indivíduo, estão armazenadas (Massad, Marin, \& Azevedo, 2003). Segundo Dick, Steen, e Detmer (1997), o Prontuário Eletrônico do Paciente (PEP) é um registro eletrônico que reside em um sistema especificamente projetado para apoiar os usuários fornecendo acesso a um completo conjunto de dados, alertas, sistemas de apoio à decisão e outros recursos, como links para bases de conhecimento médico.

De acordo com Santos (2011), o PEP envolve apenas um estabelecimento médico, um único software de apoio à gestão das informações médicas. Já o RES (Registro Eletrônico de Saúde), além de ser composto pelas informações de saúde de um paciente, é elaborado a partir de eventos ocorridos em múltiplas organizações de saúde. Assim, o RES disponibiliza informações de forma integrada de um determinado paciente. O HIMSS (Healthcare Information and Management Systems Society) apresenta a seguinte definição para o RES:

registro eletrônico longitudinal de informações de saúde do paciente gerado por um ou mais encontros em qualquer ambiente de prestação de cuidados. São incluídos dados demográficos do paciente, notas de evolução do paciente, problemas, medicamentos, sinais vitais, histórico médico, imunizações, dados laboratoriais e relatórios de radiologia" (HIMSS, 2003, tradução nossa).

Ainda segundo o HIMSS, o RES busca simplificar e automatizar o fluxo de trabalho do médico e tem a capacidade de gerar o registro completo de um encontro clínico do paciente, dando apoio também a outras atividades relacionadas ao cuidado, direta ou indiretamente, incluindo suporte com base em evidências para decisão e gestão da qualidade.

Apesar de serem sistemas importantes e benéficos para os envolvidos, existem vários desafios para se desenvolver RES (Pessanha \& Bax, 2015). Além disso, são inúmeros requisitos necessários para que eles atendam de maneira eficiente aos contextos envolvidos. A seguir são apresentadas propriedades importantes levantadas desse tipo de sistema.

\section{AS PROPRIEDADES DE SISTEMAS DE INFORMAÇÃO EM SAÚDE}

Os Sistemas Eletrônicos de Informação em Saúde são necessários e possuem características específicas, visto que armazenam dados relevantes sobre o estado de saúde e doença e se comprometem com a confidencialidade e privacidade dos mesmos e suas implicações na vida das pessoas. Eles apresentam inúmeros requisitos, tanto funcionais quanto não funcionais que precisam ser atendidos (Busato, 2015). Assim, visando documentar propriedades e possibilitar uma verificação em relação à requisitos obrigatórios para esse tipo de sistema, a Sociedade Brasileira de Informática em Saúde (SBIS) criou um processo de certificação de S-RES, em parceria com o Conselho Federal de Medicina. As normas criadas estão presentes em um manual desenvolvido: Manual de Certificação para Sistemas de Registro Eletrônico em Saúde (S-RES) (SBIS, 2013). No manual são apresentados três grandes grupos de requisitos: Segurança; Gerenciamento eletrônico de documentos; Estrutura, Conteúdo e Funcionalidades. Os requisitos de segurança são fundamentais para garantir a privacidade, confidencialidade e integridade da informação identificada em saúde. Os requisitos de Gerenciamento Eletrônico de Documentos contemplam as necessidades básicas a este tipo de recurso para a digitalização, guarda e manuseio dos prontuários em meio eletrônico. Já os requisitos de Estrutura, Conteúdo e Funcionalidades envolvem as funcionalidades que devem estar disponíveis e como os dados devem ser apresentados e armazenados.

Nesse trabalho, o foco será dado para os requisitos de Estrutura e Conteúdo, onde as propriedades levantadas estão inseridas, destacando como os dados devem ser apresentados e armazenados. Neste contexto, foram levantadas neste trabalho algumas diretrizes a serem atendidas por um S-RES:

a) atender as especificidades dos inúmeros conceitos clínicos das especialidades médicas;

b) adaptar-se às necessidades assistenciais nos vários níveis de atenção à saúde;

c) permitir que o profissional de saúde planeje as interfaces segundo suas demandas, em linguagem de fácil entendimento, sem necessidade de conhecimentos avançados em informática e nem do padrão adotado;

d) ser baseado em uma padronização internacional de registro clínico (aqui selecionada a Norma ISO 13606, que apresenta uma arquitetura de dois níveis: de informação e conhecimento.)

Vale ressaltar que a Norma ISO13606 foi selecionada para ilustrar a propriedade de padronização, visto que, no Brasil, a Portaria 2.073 do Ministério da Saúde de 31 de agosto de 2011 "regulamenta o uso de padrões de interoperabilidade e informação em saúde para sistemas de informação em saúde”. Segundo a portaria, para a definição do Registro Eletrônico em Saúde (RES) será utilizado o modelo de referência OpenEHR e para a interoperabilidade de modelos de conhecimento, incluindo arquétipos, templates e metodologia de gestão, será utilizado o padrão ISO 13606. 
De forma a ilustrar o sistema desejado, que siga as diretrizes sugeridas, o Sistema de Informação de Saúde deve atender ao seguinte cenário criado:

\begin{abstract}
Antônio é um médico que não consegue encontrar um sistema computacional que seja adequado para apoiá-lo com o registro de dados clínicos durante seu trabalho diário. Ele gostaria que fosse possivel fazer adaptações no mesmo para que o sistema lhe atendesse de maneira mais satisfatória, eficiente e eficaz, mas não tem conhecimentos suficientes em computação e não está disposto a adquirir esses conhecimentos. Assim, Antônio gostaria de definir melhor ou até adaptar os documentos, conceitos e fluxos que estão presentes no sistema. Ele pretende ainda extrair informações por meio de relatórios detalhados de suas atividades e utilizar o sistema nos vários níveis de atendimento em que atua: primário, secundário e terciário. Além disso, ele gostaria de ter a possibilidade de importar ou exportar informações dos seus pacientes com outros sistemas.
\end{abstract}

Baseado neste cenário destacam-se algumas propriedades essenciais para esse tipo de sistema.

\title{
Flexibilidade
}

Flexibilidade muitas vezes vem associada ao conceito de customização e ao de eficiência. Isso porque o usuário pode ter a possibilidade de realizar alterações que facilitam e agilizam seu trabalho. Nielsen e Molich (1990) definem em seu trabalho um conjunto de heurísticas a serem usadas em avaliações de interface, sendo uma das suas heurísticas "Flexibilidade e Eficiência", que aborda que a interface deve adaptar-se ao contexto e às necessidades do usuário.

Essa heurística também pode ser abordada como "Flexibilidade e Customização" como foi proposta em Zahabi et al. (2015). Nesse artigo, a revisão na literatura buscou por trabalhos relacionados à aspectos de usabilidade e segurança em sistemas de registros médicos e os autores propõem um conjunto de diretrizes para interfaces de sistemas médicos com foco no diagnóstico e documentação dos processos. Baseados nas revisões realizadas, a flexibilidade se mostrou como uma questão crítica. Os resultados revelam que a possibilidade de mudar o conteúdo de acordo com necessidades específicas (como formulários flexíveis) pode ser tratada como um problema de falta de recursos de customização.

Outros trabalhos apresentam a falta de flexibilidade como um problema. Gibbons, Lowry, e Patterson (2014) propuseram que os usuários querem ver diferentes pontos de vista de informações do paciente com base no papel que desempenham. Por exemplo, um dado que seja importante para um médico pode não ser crítico para outro especialista. Ou seja, cada especialidade tem suas próprias necessidades e, com isso, formulários de entradas de dados devem ser diferentes.

A flexibilidade nos sistemas também envolve a interação dos usuários com mecanismos de customização presentes na interface. Nesse contexto, o desenvolvimento por usuários finais (EUD - End User Development) pode ser definido como um conjunto de métodos, técnicas e ferramentas que permitem que os usuários dos sistemas atuem como desenvolvedores não profissionais de software e em algum ponto possam criar, modificar ou estender o sistema (Lieberman, Paternò, Klann, \& Wulf, 2006). As soluções em EUD variam de forma a oferecer aos usuários desde oportunidades de customizar os sistemas até mecanismos de reprogramação de componentes (de Souza \& Barbosa, 2006; Fischer, 2007).

\section{Padronização e Estrutura}

Abordar estrutura também evolve citar padronização, visto que esses conceitos estão relacionados entre si. De acordo com a ABNT ISO/TR 20514, os Sistemas de Registro Eletrônico são "Sistema para registro, recuperação e manipulação das informações de um Registro Eletrônico em Saúde" e devem incorporar um modelo de referência da informação em saúde. Os modelos de referência apresentam padrões para armazenamento e troca de informações entre sistemas. A padronização dos sistemas de saúde atende a diversos requisitos, mas podemos destacar (Costa, 2005):

a) Integrar uma diversidade de fontes, termos e conceitos médicos; implementados em plataformas de software e hardware distintas;

b) Facilitar a recuperação e a comunicação de informações;

c) Propiciar o uso de sistemas de apoio a tomadas de decisões.

Existem diferentes padrões que podem ser adotados para o desenvolvimento de RES. Os aqui abordados (OpenEHR e ISO13606), como já citado, foram os adotados baseados na portaria 2.073 do Ministério da Saúde. A Fundação OpenEHR ${ }^{3}$ tem como objetivo tornar realidade o registro eletrônico de saúde algo duradouro e melhorar a qualidade dos cuidados com a saúde nos sistemas de informação. O padrão OpenEHR consiste em uma especificação aberta para a construção de sistemas de RES, influenciada pelos resultados de projetos na

${ }^{3}$ http://www.openehr.org/pt/about/foundation.php. 
União Europeia e Austrália, desde o início dos anos 1990 (Santos, 2011). A ISO13606 aborda a comunicação entre registros eletrônicos de saúde (Health Informatics - Eletronic health record communication).

\section{Facilidade de interação}

No processo de interação usuário-sistema de informação, a interface é o combinado de software e hardware necessário para viabilizar e facilitar os processos de comunicação entre o usuário e a aplicação (Preece et al., 1994). Segundo Moran (1981), a interface de usuário deve ser entendida como sendo a parte de um sistema computacional com a qual uma pessoa entra em contato de forma física, perceptiva e conceitual. O termo Interação Humano-Computador (IHC) foi adotado na década de 1980 para descrever um novo campo de estudo. $\mathrm{O}$ termo não abrange apenas interfaces, mas todos os aspectos relacionados à interação entre pessoas e sistemas computacionais (Preece et al., 1994). Trata-se de uma matéria multidisciplinar que relaciona ciência da computação, design, ergonomia, psicologia, sociologia, semiótica, linguística e áreas afins.

Os objetivos de IHC podem ser resumidos em "desenvolver ou melhorar a segurança, utilidade, eficácia, eficiência e usabilidade de sistemas computacionais” (Barlow, Rada, \& Diaper, 1989). O termo “Sistemas” aqui não está se referindo a software ou hardware especificamente, mas todo o contexto de uso. Utilidade, portanto, se refere às funcionalidades do sistema. A eficácia relaciona-se com a precisão, completeza com que os usuários atingem objetivos específicos, acessando a informação correta ou gerando os resultados esperados, enquanto a eficiência está relacionada com a precisão, completeza com que os usuários atingem seus objetivos em relação à quantidade de recursos gastos. Usabilidade envolve a premissa de que o sistema deve ser fácil de aprender $\mathrm{e}$ fácil de usar (Barbosa \& Silva, 2010; Preece et al., 1994).

\section{ANÁLISE DOS SISTEMAS DE REGISTOS ELETRÔNICOS DE SAÚDE}

A etapa de análise e avaliação das soluções existentes envolveu em um primeiro momento selecionar os sistemas a serem avaliados. Neste caso, podemos abordar os ambientes acadêmico, comercial e governamental; isso porque os sistemas são desenvolvidos para serem utilizados em diversos tipos de instituições: públicas, privadas, clínicas, hospitais, centros de atendimento etc. Todos possuem visões específicas do problema, mas também compartilham preocupações com o tema. De forma geral, todas as instituições buscam criar sistemas que possam tornam a vida dos pacientes e dos profissionais mais agradável e que permitam que as informações sejam armazenadas de forma segura e completa.

Em todos os ambientes existe o foco de se criar sistemas que sejam fáceis de serem utilizados, eficientes e adequados para o contexto. No ambiente comercial há, ainda, a preocupação para que o sistema tenha um custo acessível ou mesmo sem custo direto para os usuários, tais como nos sistemas CommuniMed ${ }^{4}$ e PracticeFusion ${ }^{5}$. O contexto governamental aborda os sistemas de registro eletrônico de saúde por diversos outros aspectos, assim como no ambiente acadêmico. Além de toda expectativa em termos de eficiência de uso e eficácia, há também o foco em padronizações visando a interoperabilidade. Nesse sentido, abordam aspectos de armazenamento dos dados, padrões usados para compartilhar informações, como tornar esses sistemas mais adequados também à população, etc. O SisMater ${ }^{6}$ (Sistema de informação em saúde Materna e Neonatal), utilizado no hospital das clínicas na UFMG, é um exemplo de sistema desenvolvido e utilizado no meio acadêmico e governamental.

Para a análise realizada nesse trabalho foram selecionados os três sistemas citados: PracticeFusion, CommuniMed e SisMater. A escolha foi feita na tentativa de se ter acesso a tipos variados de sistemas, sendo os três acessíveis pela web. No caso do PracticeFusion, trata-se de um sistema gratuito, aberto, com significativo número de usuários (tal como apresentado pelo sistema) ${ }^{7}$. O CommuniMed e SisMater são sistemas "fechados" mas que puderam ser analisados devido o envolvido dos autores com os mesmos. Dessa forma, para todos eles foram criados usuários de teste que permitiram a interação e análise. O CommuniMed consiste em um sistema de prontuário médico que se apresenta da seguinte forma: "O CommuniMed é um sistema de prontuário eletrônico online multi-especialidade focado em usabilidade [...]”. O Pratice Fusion é uma plataforma de EHR para médicos e pacientes. É apresentada como "a plataforma número 1 de registro eletrônico em saúde baseada nas nuvens para médicos e pacientes”. O SisMater, desde de 2012, é o sistema de registro de saúde utilizado com foco na saúde materno-infantil já em uso no Hospital das Clínicas da UFMG. Vale ressaltar que consiste em um sistema que atende ao setor terciário de saúde, documentando as intervenções feitas no Hospital. O perfil de usuário principal do sistema consiste nos médicos obstetras e pediatras responsáveis pelo cuidado e atendimento de mulheres grávidas em internações ante parto, com parto e pós-parto.

\footnotetext{
${ }^{4} \mathrm{http}: / /$ www.communimed.com.br/.

${ }^{5} \mathrm{http}: / /$ www.practicefusion.com/.

${ }^{6} \mathrm{http}: / /$ sismater.hc.ufmg.br/.

${ }^{7}$ Segundo consta no site da empresa, o sistema tem significativo número de usuários e seu atrativo é a total gratuidade no uso. (http://www.practicefusion.com/).
} 


\section{Questões de análise}

Um sistema pode ser analisado e avaliado por diferentes perspectivas e em áreas distintas, tais como interface, segurança, desempenho etc. No contexto de IHC, por exemplo, as avaliações consistem em momentos onde é possível analisar a solução proposta (seja parcial ou final), identificar problemas e analisá-la de forma a propor outros caminhos, se necessário (Barbosa \& Silva, 2010). Nesse caso, o foco consiste nos problemas encontrados na interação com o sistema. Em cada análise é feita uma seleção de quais critérios serão avaliados, ou seja, quais serão os pontos analisados em um determinado sistema.

Os métodos de avaliação podem ser classificados em métodos de investigação, de inspeção e de observação. Os de investigação envolvem questionários, entrevistas, estudos de campos, técnicas que permitem que o avaliador analise os dados coletados. Nos métodos de inspeção, cabe ao avaliador, especialista na área, utilizar, analisar e avaliar o sistema de acordo com os propósitos do método escolhido. Os métodos de observação envolvem a análise do sistema sendo utilizado em ambientes reais, coletando eventos que ocorrem no dia a dia, durante a execução de tarefas.

Para análise aqui apresentada utilizou-se o método de inspeção, visto que as questões (que indicam os critérios) foram disponibilizadas para dois especialistas que procederam a análise. Vale ressaltar que tais questões foram baseadas nas propriedades de Flexibilidade, Padronização e Estrutura e Facilidade de interação (Quadro 1).

\begin{tabular}{|ll|}
\hline Propriedades & Questões de análise \\
\hline Flexibilidade & - O sistema permite usar diferentes visões de acordo com o papel desempenhado \\
& pelo usuário, compartilhando informações? \\
& - O usuário pode realizar alterações nos formulários de entrada de dados, personali- \\
& zando o que considerar necessário? \\
\hline Padronização e Estrutura & - O sistema é baseado em alguma padronização dos dados? (como a ISO13606 ou \\
& OpenEHR) \\
& - É possível realizar consultas e gerar relatórios baseados nas entradas estruturadas \\
& de dados? \\
\hline Facilidade de interação & - O usuário consegue realizar seu fluxo de trabalho de forma clara? \\
& - Os símbolos e termos utilizados são próximos da realidade dos usuários? \\
\hline
\end{tabular}

Quadro 1. Questões de análise para avaliação dos sistemas de RES.

Fonte: os autores (2016).

Baseados nas questões apresentadas, uma análise foi feita dos sistemas selecionados, apresentada a seguir.

\section{Análise propriamente dita}

A partir de cada propriedade e questões de análise, os sistemas foram analisados por dois especialistas em avaliações de sistemas e os resultados são apresentados no Quadro 2.

A Figura 2 apresenta telas dos sistemas analisados que ilustram aspectos apresentados no Quadro 2.

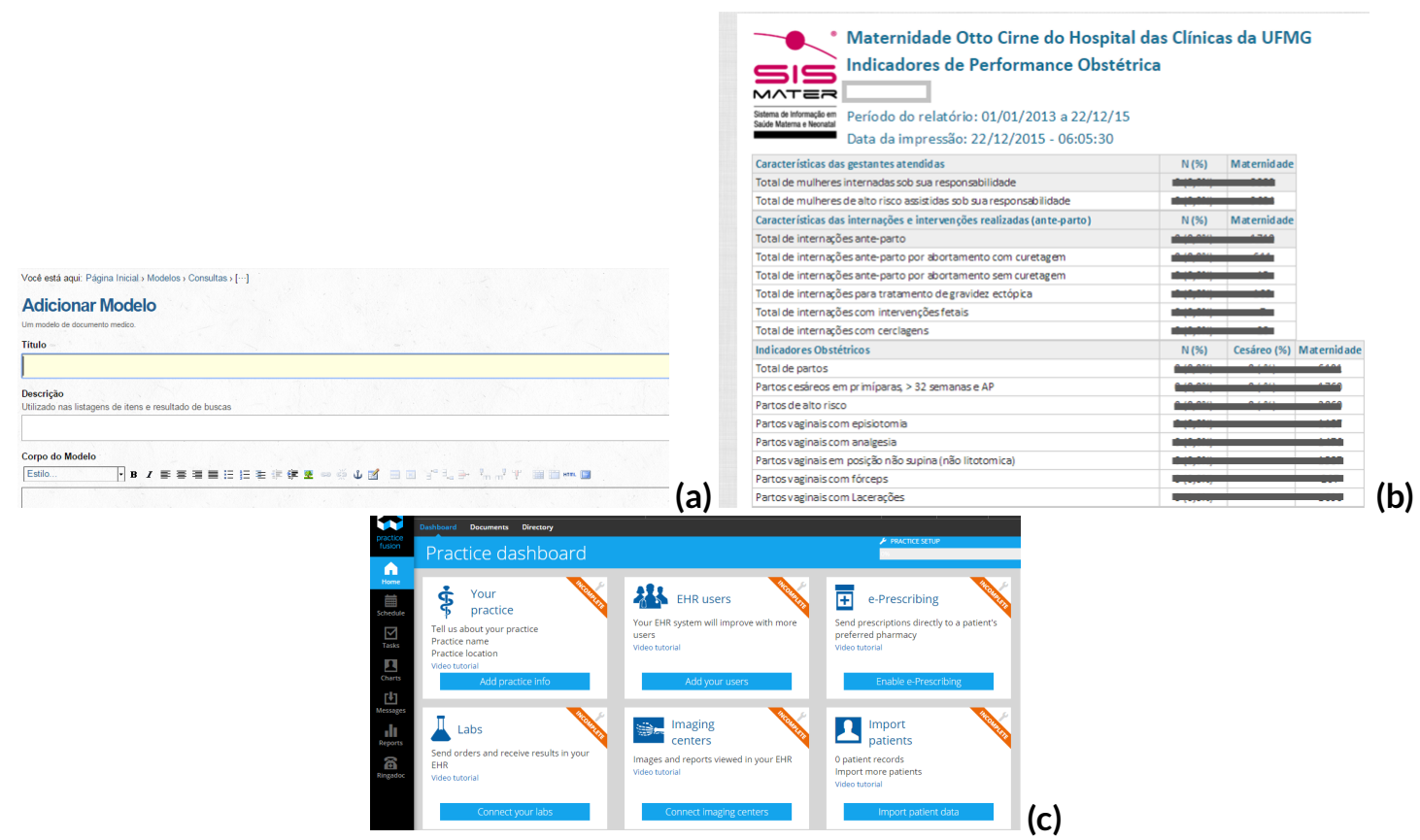

Figura 2. (a) Tela do CommuniMed - Recursos para criação de modelo de documento. (b) Tela do SisMater - Exemplo de relatório consolidado gerado no Sistema. (c) Tela do Practice Fusion - Etapas a serem configuradas no Sistema, pelos usuários.

Fonte: (a) Sistema CommuniMed. (b) Sistema SisMater. (c) Sistema Practice Fusion. 


\begin{tabular}{|c|c|c|}
\hline Propriedade & Sistema & Questões de análise respondidas \\
\hline \multirow[t]{3}{*}{ Flexibilidade } & CommuniMed & $\begin{array}{l}\text { - O sistema possui interfaces diferentes para dois perfis distintos: médicos e secre } \\
\text { tárias. Esses dois perfis conseguem compartilhar algumas informações, mas não é } \\
\text { possível que dois médicos, de especialidades iguais ou não, compartilhem dados ou } \\
\text { formulários. } \\
\text { - O usuário pode gerenciar modelos de formulários de entrada de dados e relatórios } \\
\text { a serem impressos. Uma questão a ser abordada nesse ponto é que os modelos } \\
\text { criados permitem que os usuários entrem com textos livres, dando maior liberdade } \\
\text { de escrita, mas perdendo em estrutura dos dados (Figura 2.a). }\end{array}$ \\
\hline & Practice Fusion & $\begin{array}{l}\text { - O sistema possui interfaces diferentes para médicos, pacientes e analistas de dados } \\
\text { O usuário pode personalizar algumas informações em sua área de trabalho. } \\
\text { - O médico pode personalizar seus formulários de entrada de dados criando novos } \\
\text { formulários e compartilhando os mesmos com outros profissionais. }\end{array}$ \\
\hline & SisMater & $\begin{array}{l}\text { - O sistema é voltado para um contexto bem definido, mas há perfis diferentes para } \\
\text { médicos e gestores. } \\
\text { - O usuário não tem liberdade de configurar novos modelos de formulários de entrada } \\
\text { de dados. }\end{array}$ \\
\hline \multirow{3}{*}{ Padronização e Estrutura } & CommuniMed & $\begin{array}{l}\text { - O sistema não é baseado nos padrões citados. } \\
\text { - O sistema ainda não oferece módulo para realização de consultas com geração de } \\
\text { relatórios. }\end{array}$ \\
\hline & Practice Fusion & $\begin{array}{l}\text { - O sistema não é baseado nos padrões citados. } \\
\text { - O sistema oferece módulo para geração de relatórios consolidados sobre os dados } \\
\text { coletados. }\end{array}$ \\
\hline & SisMater & $\begin{array}{l}\text { - O sistema não é baseado nos padrões citados. } \\
\text { - O sistema oferece sumarização de dados das atividades realizadas e relatórios } \\
\text { gerais de desempenho do profissional (Figura 2.b). }\end{array}$ \\
\hline \multirow{3}{*}{ Facilidade de interação } & CommuniMed & $\begin{array}{l}\text { - Os símbolos e termos utilizados estão de acordo com o perfil dos usuários. } \\
\text { - O fluxo de trabalho dos médicos envolvidos pode ser claramente seguido usando os } \\
\text { recursos disponíveis. }\end{array}$ \\
\hline & Practice Fusion & $\begin{array}{l}\text { - O sistema apresenta diferentes símbolos e termos, normalmente comuns para os } \\
\text { usuários, mas alguns podem se sentir confusos com a variedade de informações } \\
\text { disponíveis. } \\
\text { - O fluxo de trabalho dos médicos envolvidos pode ser realizado mas existem várias } \\
\text { etapas a serem seguidas e configuradas (Figura 2.c), o que pode causar dúvidas aos } \\
\text { usuários. }\end{array}$ \\
\hline & SisMater & $\begin{array}{l}\text { - Os símbolos e termos utilizados estão de acordo com o perfil dos usuários. } \\
\text { - O fluxo de trabalho dos médicos envolvidos pode ser claramente seguido usando os } \\
\text { recursos disponíveis. }\end{array}$ \\
\hline
\end{tabular}

Quadro 2. Análises realizadas nos sistemas (conforme orientação do Quadro 1).

Fonte: os autores com base em dados da pesquisa (2016).

No Quadro 3 verifica-se que nenhum dos sistemas analisados aborda todas as propriedades de forma conjunta.

\begin{tabular}{|llll|}
\hline & CommuniMed & Practice Fusion & SisMater \\
\hline Flexibilidade & $\begin{array}{l}\text { Flexibilidade na criação de do- } \\
\text { cumentos mas falta compartilha- } \\
\text { mento de dados. }\end{array}$ & $\begin{array}{l}\text { Flexibilidade na criação de docu- } \\
\text { mentos e compartilhamento par- } \\
\text { cial de dados. }\end{array}$ & $\begin{array}{l}\text { Falta de flexibilidade na criação } \\
\text { de documentos e compartilha- } \\
\text { mento parcial de dados. }\end{array}$ \\
\hline Padronização e Estrutura & $\begin{array}{l}\text { Não é baseado nos padrões cita- } \\
\text { dos e consolidação parcial dos da- } \\
\text { dos. }\end{array}$ & $\begin{array}{l}\text { Não é baseado nos padrões cita- } \\
\text { dos mas há consolidação dos da- } \\
\text { dos. }\end{array}$ & $\begin{array}{l}\text { Não é baseado nos padrões cita- } \\
\text { dos mas há consolidação dos da- } \\
\text { dos. }\end{array}$ \\
\hline Facilidade de interação & $\begin{array}{l}\text { Símbolos e fluxo de trabalho ade-- } \\
\text { quados aos usuários. }\end{array}$ & $\begin{array}{l}\text { Símbolos e fluxo de trabalho po- } \\
\text { dem ser complexos para os usuá- } \\
\text { rios. }\end{array}$ & $\begin{array}{l}\text { Símbolos e fluxo de trabalho ade- } \\
\text { quados aos usuários. }\end{array}$ \\
& & & \\
\hline
\end{tabular}

Quadro 3. Resumo das análises.

Fonte: os autores (2016).

Dois sistemas analisados apresentam certa flexibilidade, mas têm limitações quanto à persistência estruturada dos conceitos e a interoperabilidade com outros sistemas (papel da padronização). Porém, as soluções em que persistem a estrutura não são flexíveis para acomodar diferenças conceituais entre especialidades ou perfis diferentes na mesma especialidade. Verifica-se que, em nenhum caso, foi possível atender às três propriedades levantadas. Trata-se de um desafio criar um sistema robusto no armazenamento e compartilhamento dos dados, mas que seja flexível em sua interface, atendendo às necessidades específicas dos usuários.

\section{CONSIDERAÇÕES FINAIS}

Os Sistemas de Registros Eletrônico em Saúde são plataformas relevantes tanto para profissionais da saúde quanto para os pacientes e, para os quais, a organização e padronização dos dados são exigências críticas. As informações de tais sistemas podem ser utilizadas para melhorar o atendimento dos profissionais e apoiar estudos voltados à toda uma população.

Criar um sistema RES/PEP que atenda aos três requisitos em foco neste artigo constitui um projeto complexo. A 
análise realizada teve como objetivo principal ilustrar o nível de dificuldade de tal projeto. Não é do conhecimento dos autores a existência hoje uma solução que apresente três características essenciais (flexibilidade, padronização e estrutura e facilidade de interação), e que contemple um padrão para troca de informações. Dessa forma, a busca contínua por soluções para esse tipo de sistema se torna necessária, pois os desafios que ocorrem não são pontuais ou específicos de um contexto de uso.

Como trabalho futuro, propõem-se a criação de um modelo de interface extensível de interface para sistemas de Registro Eletrônico de Saúde baseados na ISO 13606. A proposta do modelo consiste em criar uma camada de abstração dos conceitos envolvidos no padrão da Norma ISO 13606, permitindo que os usuários possam reutilizar, customizar e compartilhar conceitos e documentos, persistindo os dados de forma padronizada. $\mathrm{O}$ objetivo é que o modelo suporte a geração de interfaces que ofereçam as três propriedades destacadas como importantes para esses sistemas. Além do modelo, um protótipo será criado e avaliado com profissionais envolvidos. 


\section{REFERÊNCIAS}

Abrahão, M. S. (2003). A segurança da informação digital na saúde. Sociedade Beneficente Israelita Brasileira.

Albergaria, E. T., Bax, M. P., \& Prates, R. O. (2013). Interação Humano Computador na Ciência da Informação. In XIV ENANCIB - Encontro Nacional de Pesquisa em Ciência da Informação. Florianópolis: Ancib; UFSC. Recuperado de http://enancib.sites.ufsc.br/index.php/enancib2013/ XIVenancib/paper/view/343

Albergaria, E. T., Bax, M. P., Prates, R. O., \& Rocha, L. . (2014a). Caracterizando desafios de interação em ferramentas de modelagem de dados clínicos. In XIV Congresso Brasileiro em Informática em Saúde. Santos.

Albergaria, E. T., Bax, M. P., Prates, R. O., \& Rocha, L. C. D. (2014b). Caracterizando os desafios na modelagem dos dados clínicos em sistemas de RES baseados no OpenEHR. In WIM - XIV Workshop de Informática Médica; XXXIV Congresso da Sociedade Brasileira de Computação. Brasília: UNB.

Alves, A. F. (2015). Implementação do OpenEHR para uma aplicação móvel de registro eletrônico de saúde: Estudo em uma empresa de saúde. In II Seminário Tecnologias Aplicadas a Educação e Saúde. UNEB. Recuperado de http://www.revistas .uneb.br/index.php/staes/article/view/1614

Barbosa, S. D. J., \& Silva, B. S. (2010). Interação humanocomputador. Campus-Elsevier.

Barlow, J., Rada, R., \& Diaper, D. (1989, April). Interacting with computers. Interacting with Computers, 1(1), 39-42. doi: 10.1016/0953-5438(89)90006-4

Bax, M. P. (2013, maio/ago.). Design science: filosofia da pesquisa em ciência da informação e tecnologia. Ciência da informação, 42(2), 298-312. Recuperado de http://revista.ibict.br/ ciinf/article/view/1388 doi: 10.18225/ci.inf..v42i2.1388

Bayer, R., Santelli, J., \& Klitzman, R. (105). New challenges for electronic health records: confidentiality and access to sensitive health information about parents and adolescents. Journal of the American Medical Association, 313(2), 29-30. doi: 10.1001/jama.2014.15391

Beale, T. (2002). Archetypes: Constraint-based domain models for future-proof information systems. In OOPSLA 2002 Workshop on Behavioural Semantics. Seattle.

Bodenreider, O. (2004). The unified medical language system (UMLS): integrating biomedical terminology. $\mathrm{Nu}$ cleic Acids Research, 32(Database issue), 267-270. doi: 10.1093/nar/gkh061

Busato, C. (2015). Funcionalidades para sistemas de registro eletrônico em saúde na atenção primária à saúde (Dissertação de Mestrado em Epidemiologia, Universidade Federal do Rio Grande do Sul, Porto Alegre, RS, Brasil). Recuperado de https://www.lume.ufrgs.br/bitstream/handle/10183/ 115081/000953386.pdf?sequence=1

Certification Commission for Health Information Technology. (2011). CCHIT Ambulatory EHR: Certification criteria. Recuperado em Dez. 2015, de http://www.cchit.org/

Clarke, M. A., Steege, L. M., Moore, J. L., Belden, J. L., Koopman, R. J., \& Kim, M. S. (2013). Addressing human computer interaction issues of electronic health record in clinical encounters. In A. Marcus (Ed.), Design, user experience, and usability: Health, learning, playing, cultural, and cross-cultural user experience (p. 381-390). Berlin, Germany: Springer.

Costa, E. (2005). Prontuário do paciente: informações para a gestão em saúde (Monografia de conclusão do Curso Técnico de Gestão nos Serviços de Saúde). Fundação Oswaldo Cruz, Rio de Janeiro, RJ, Brasil.

de Souza, C. S., \& Barbosa, S. D. J. (2006). A semiotic framing for end-user development. In H. Lieberman, F. Paternò, \& V. Wulf (Eds.), End user development (p. 401-426). New York, USA: Springer.

Detmer, D., Bloomrosen, M., Raymond, B., \& Tang, P. (2008, Oct.). Integrated personal health records: Transformative tools for consumer-centric care. BMC Medical Informatics and Decision Making, 8(45). doi: 10.1186/1472-6947-8-45

Dick, R. S., Steen, E. B., \& Detmer, D. E. (1997). The computer-based patient record: An essential technology for health care, revised edition. The National Academies Press. doi: $10.17226 / 5306$

Fischer, G. (2007). Meta-design: Expanding boundaries and redistributing control in design. In C. Baranauskas, P. Palanque, J. Abascal, \& S. D. J. Barbosa (Eds.), Human-computer interaction - interact 2007: 11th ifip tc 13 international conference, rio de janeiro, brazil, september 10-14, 2007, proceedings, part $i$ (p. 193-206). Berlin, Heidelberg: Springer Berlin Heidelberg. doi: 10.1007/978-3-540-74796-3_19

Gibbons, M. C., Lowry, S. Z., \& Patterson, E. S. (2014). Applying human factors principles to mitigate usability issues related to embedded assumptions in health information technology design. JMIR Human Factors, 1(1). doi: 10.2196/humanfactors. 3524

Healthcare Information and Management Systems Society. (2003). HIMSS eletronic health record definitional model: Version 1.1. openclinical: Electronic medical records. Recuperado em Dez. 2015, de http://www.openclinical.org/emr.html\# HIMSS2003

Hillestad, R., Bigelow, J., Bower, A., Girosi, F., Meili, R., Scoville, R., \& Taylor, R. (2005, Sep.). Can electronic medical record systems transform health care? potential health benefits, savings, and costs. Health Affairs, 24(5), 1103-1117. doi: 10.1377/hlthaff.24.5.1103

International Organization for Standardization. (2005). ISO/TR 20.514:2005. Health Informatics - Electronic health record - Definition, scope and context. Recuperado de http://www.iso.org/iso/iso_catalogue/catalogue_tc/ catalogue_detail.htm?csnumber=39525

Lieberman, H., Paternò, F., Klann, M., \& Wulf, V. (2006). Enduser development: An emerging paradigm. In H. Lieberman, F. Paternò, \& V. Wulf (Eds.), End user development. Dordrecht: Springer Netherlands. doi: 10.1007/1-4020-5386-X_1

Maia, T. A. (2015). Processo de desenvolvimento de arquétipos do registro eletrônico em saúde em minas gerais: Estudo de caso (Dissertação de Mestrado Profissional em Sistemas de Informação e Gestão do Conhecimento, Universidade FUMEC, Belo Horizonte, MG, Brasil). Recuperado de http://www.fumec.br/revistas/sigc/article/view/2685/1620

Marin, H. F., \& Azevedo, R. S. (2003). O prontuário eletrônico do paciente na assistência, informação e conhecimento médico. São Paulo: H. de F. Marin.

Massad, E., Marin, H. F., \& Azevedo, R. S. (2003). O prontuário eletrônico do paciente na assistência, informação e conhecimento médico. São Paulo: H. de F. Marin.

Moran, T. P. (1981, July). The command language grammar: a representation for the user interface of interactive computer systems. International Journal of Man-Machine Studies, 15(1), 3-50. doi: 10.1016/S0020-7373(81)80022-3

Nielsen, J., \& Molich, R. (1990). Heuristic evaluation of user 
interfaces. In SIGCHI Conference on Human Factors in Computing Systems (p. 249-256). ACM. doi: 10.1145/97243.97281

Pessanha, C. P., \& Bax, M. P. (2015). Implementando o prontuário eletrônico OpenEHR em sistemas gestores de conteúdo: uma aproximação. In XVI ENANCIB - Encontro Nacional de Pesquisa em Ciência da Informação. João Pessoa: Ancib; UFPB. Recuperado de http://www.ufpb.br/evento/lti/ ocs/index.php/enancib2015/enancib2015/paper/view/2756

Preece, J., Rogers, Y., Sharp, H., Benyon, D., Holland, S., \& Carey, T. (1994). Human computer interaction. England: Addison Wesley.

Ratwani, R. M., Fairbanks, R. J., Hettinger, A. Z., \& Benda, N. C. (2015). Electronic health record usability: analysis of the user-centered design processes of eleven electronic health record vendors. Journal of the American Medical Informatics Association, 22(6), 1179-1182. doi: 10.1093/jamia/ocv050

Santos, M. (2011). Sistema de registro eletrônico de saúde baseado na norma ISO 13606: Aplicações na Secretaria de Saúde de Estado de Minas Gerais (Tese de Doutorado em Ciência da Informação, Universidade Federal de Minas Gerais, Belo Horizonte). Recuperado de http://www.bibliotecadigital.ufmg.br/dspace/bitstream/ handle/1843/ECIC-8L8HFJ/tese_eci_ufmg__marcelo _rodrigues_dos_santos_2011.pdf?sequence $=1$

Simon, H. A. (1996). The sciences of the artificial (3a. ed.). USA: MIT Press.

Sociedade Brasileira de Informática em Saúde. (2013). Manual de certificação para sistemas de Registro Eletrônico em Saúde (S-RES): versão 4.1. Recuperado de http://www.sbis.org.br/certificacao/Manual_Certificacao _SBIS-CFM_2013_v4-1.pdf

Spooner, S. A. (2007). Special requirements of electronic health record systems in pediatrics. Pediatrics, 119(3), 631637. Recuperado de http://pediatrics.aappublications.org/ content/119/3/631

Wieringa, R. (2009). Design science as nested problem solving. In Proceedings of the 4th international conference on design science research in information systems and technology (p. 8:1-8:12). New York, NY, USA: ACM. doi: $10.1145 / 1555619.1555630$

Zahabi, M., Kaber, D. B., \& Swangnetr, M. (2015, Aug.). Usability and safety in electronic medical records interface design: A review of recent literature and guideline formulation. Human Factors, 57(8), 805-834. doi: $10.1177 / 0018720815576827$

Como citar este artigo (APA):

Albergaria, E. T., Bax, M. P., Prates, R. O. \& Reis, Z. S. N. (2016). Identificando propriedades essenciais de registros eletrônicos de saúde. AtoZ: novas práticas em informação e conhecimento, 5(1), 33 - 43. Recuperado de: http://dx.doi.org/10.5380/atoz.v5i1.44738 